\title{
A SIGN PRESERVING MIXED FINITE ELEMENT APPROXIMATION FOR CONTACT PROBLEMS
}

\author{
PATRICK HILD \\ Besançon Laboratory of Mathematics, UMR CNRS 6623 \\ Franche-Comté University, 16 route de Gray, 25030 Besançon, France \\ e-mail: patrick.hild@univ-fcomte.fr
}

\begin{abstract}
This paper is concerned with the frictionless unilateral contact problem (i.e., a Signorini problem with the elasticity operator). We consider a mixed finite element method in which the unknowns are the displacement field and the contact pressure. The particularity of the method is that it furnishes a normal displacement field and a contact pressure satisfying the sign conditions of the continuous problem. The a priori error analysis of the method is closely linked with the study of a specific positivity preserving operator of averaging type which differs from the one of Chen and Nochetto. We show that this method is convergent and satisfies the same a priori error estimates as the standard approach in which the approximated contact pressure satisfies only a weak sign condition. Finally we perform some computations to illustrate and compare the sign preserving method with the standard approach.
\end{abstract}

Keywords: variational inequality, positive operator, averaging operator, contact problem, Signorini problem, mixed finite element method.

\section{Introduction}

Finite element methods are efficient and widespread tools in computational contact and impact mechanics (see Han and Sofonea, 2002; Haslinger et al., 1996; Kikuchi and Oden, 1988; Laursen, 2002; Wriggers, 2002), and mixed formulations involving a displacement field $\boldsymbol{u}$ in the bodies and the contact pressure $\sigma_{n}(\boldsymbol{u})$ on the contact zone are commonly used. A particularity of the contact problem lies in the so-called unilateral conditions linking on the contact zone $\Gamma_{C}$, the normal displacement field $u_{n}$ and the Lagrange multiplier $\lambda=-\sigma_{n}(\boldsymbol{u})$ :

$$
u_{n} \leq 0, \quad \lambda \geq 0, \quad \lambda u_{n}=0 \quad \text { on } \Gamma_{C} .
$$

The mixed finite element method we consider, introduced by Hild and Nicaise (2007), furnishes an approximated normal displacement field $u_{h n}$ and an approximated multiplier $\lambda_{h}$ which satisfy

$$
\begin{array}{cc}
u_{h n} \leq 0, & \lambda_{h} \geq 0 \quad \text { on } \Gamma_{C}, \\
\lambda_{h} u_{h n}=0 & \text { at the nodes of } \Gamma_{C} .
\end{array}
$$

Such a method shows three interesting aspects in comparison with the standard approach in which the multiplier is only nonnegative in a weak sense (see, e.g., Ben Bel- gacem and Renard, 2003; Coorevits et al., 2002; Hüeber and Wohlmuth, 2005a):

- The nonnegative multiplier is more relevant from a mechanical point of view.

- This multiplier vanishes where the body separates (the multiplier of the standard approach may reveal some artificial oscillations in the separation zone).

- It allows defining a simple a posteriori error estimator whose numerical analysis gives better bounds than for the error estimator arising from the standard approach (see Hild and Nicaise, 2007).

Let us mention that there exist other mixed formulations leading to a priori error estimates with nonnegative multipliers and normal displacement fields which do not satisfy the nonpositivity condition (see Ben Belgacem and Brenner, 2001; Ben Belgacem and Renard, 2003; Haslinger et al., 1996).

The paper is organized as follows. In Section 2 we introduce the equations modelling the frictionless unilateral contact problem between an elastic body and a rigid foundation. We write the problem using a formulation where the unknowns are the displacement field in the body and 
the pressure on the contact area. In Section 3, we choose a discretization involving continuous finite elements of degree 1 for the displacements and continuous piecewise affine multipliers on the contact zone. The main particularity of this approach is that both the normal displacement and the multiplier solution to the discrete problem satisfy the same sign conditions as the normal displacement and the multiplier solving the continuous problem.

More precisely, the displacement field of the sign preserving method coincides with the one in the standard approach, and the multipliers are linked by a linear operator which transforms the functions satisfying some "weak" nonnegativity conditions into nonnegative functions. In Section 4, we study and discuss the main basic properties of the positivity preserving averaging operator which requires minimal regularity. Section 5 is concerned with the a priori error analysis of the sign preserving method. We prove that the method is convergent when using convenient regularity assumptions on the solution to the continuous problem, and we obtain similar a priori error estimates as for the standard approach. In Section 6 we implement both methods and compare them using several examples. As expected, the sign preserving method furnishes more relevant multipliers and no loss of convergence is observed in comparison with the standard approach. Finally, we mention that the results in this paper obviously hold for the simpler Signorini problem with the Laplace operator.

As usual, we denote by $\left(H^{s}(\cdot)\right)^{d}, s \in \mathbb{R}, d=1,2$ the Sobolev spaces in one and two space dimensions (see Adams, 1975). The usual norm of $\left(H^{s}(D)\right)^{d}$ (dual norm if $s<0)$ is denoted by $\|\cdot\|_{s, D}$, and we keep the same notation when $d=1$ or $d=2$.

\section{Unilateral contact problem in linear elasticity}

We consider an elastic body $\Omega$ in $\mathbb{R}^{2}$ where plane strain assumptions are made. The boundary $\partial \Omega$ of $\Omega$ is polygonal, and we suppose that $\partial \Omega$ consists of three nonoverlapping parts $\Gamma_{D}, \Gamma_{N}$ and $\Gamma_{C}$ with meas $\left(\Gamma_{D}\right)>0$ and meas $\left(\Gamma_{C}\right)>0$. The normal unit outward vector on $\partial \Omega$ is denoted by $\boldsymbol{n}=\left(n_{1}, n_{2}\right)$, and we choose as the unit tangential vector $\boldsymbol{t}=\left(-n_{2}, n_{1}\right)$. In its initial stage, the body is in contact on $\Gamma_{C}$ which is supposed to be a straight line segment, and we suppose that the unknown final contact zone after deformation will be included in $\Gamma_{C}$. The body is clamped on $\Gamma_{D}$ for the sake of simplicity. It is subjected to volume forces $\boldsymbol{f}=\left(f_{1}, f_{2}\right) \in\left(L^{2}(\Omega)\right)^{2}$ and surface loads $\boldsymbol{g}=\left(g_{1}, g_{2}\right) \in\left(L^{2}\left(\Gamma_{N}\right)\right)^{2}$.

The unilateral contact problem in linear elasticity consists in finding a displacement field $u: \Omega \rightarrow \mathbb{R}^{2}$ satisfying the equations and conditions (1)-(6):

$$
\operatorname{div} \sigma(\boldsymbol{u})+\boldsymbol{f}=\mathbf{0} \quad \text { in } \Omega,
$$

where $\boldsymbol{\sigma}=\left(\sigma_{i j}\right), \quad 1 \leq i, j \leq 2$, stands for the stress tensor field and $\boldsymbol{d i v}$ denotes the divergence operator of tensor valued functions. The stress tensor field is obtained from the displacement field by the constitutive law of linear elasticity:

$$
\boldsymbol{\sigma}(\boldsymbol{u})=\boldsymbol{A} \varepsilon(\boldsymbol{u}) \quad \text { in } \Omega,
$$

where $\boldsymbol{A}$ is a fourth order symmetric and elliptic tensor whose coefficients lie in $C^{1}(\bar{\Omega})$, and $\varepsilon(\boldsymbol{v})=\left(\nabla \boldsymbol{v}+{ }^{t}\right.$ $\nabla \boldsymbol{v}) / 2$ represents the linearized strain tensor field. On $\Gamma_{D}$ and $\Gamma_{N}$, the conditions are as follows:

$$
\begin{aligned}
& \boldsymbol{u}=\mathbf{0} \quad \text { on } \Gamma_{D} \\
& \boldsymbol{\sigma}(\boldsymbol{u}) \boldsymbol{n}=\boldsymbol{g} \quad \text { on } \Gamma_{N} \text {. }
\end{aligned}
$$

For any displacement field $\boldsymbol{v}$ and for any density of surface forces $\boldsymbol{\sigma}(\boldsymbol{v}) \boldsymbol{n}$ defined on $\partial \Omega$, we adopt the following notation:

$$
\boldsymbol{v}=v_{n} \boldsymbol{n}+v_{t} \boldsymbol{t}, \quad \boldsymbol{\sigma}(\boldsymbol{v}) \boldsymbol{n}=\sigma_{n}(\boldsymbol{v}) \boldsymbol{n}+\sigma_{t}(\boldsymbol{v}) \boldsymbol{t} .
$$

The three conditions describing unilateral contact on $\Gamma_{C}$ are (see, e.g., Duvaut and Lions, 1972; Eck et al., 2005; Fichera, 1964; 1972)

$$
u_{n} \leq 0, \quad \sigma_{n}(\boldsymbol{u}) \leq 0, \quad \sigma_{n}(\boldsymbol{u}) u_{n}=0 .
$$

Finally, the equality

$$
\sigma_{t}(\boldsymbol{u})=0
$$

on $\Gamma_{C}$ means that friction is omitted.

The mixed variational formulation of (1)-(6) uses the Hilbert space

$$
\boldsymbol{V}=\left\{\boldsymbol{v} \in\left(H^{1}(\Omega)\right)^{2}: \quad \boldsymbol{v}=\mathbf{0} \text { on } \Gamma_{D}\right\} .
$$

The Lagrange multiplier space $M$ is the dual of the normal trace space $N$ of $\boldsymbol{V}$ restricted to $\Gamma_{C}$. If the end points of $\Gamma_{C}$ belong to $\bar{\Gamma}_{N}$ (resp. $\bar{\Gamma}_{D}$ ), then $N=H^{\frac{1}{2}}\left(\Gamma_{C}\right)$ (resp. $H_{00}^{\frac{1}{2}}\left(\Gamma_{C}\right)$ ). We next define the following convex cone of multipliers on $\Gamma_{C}$ :

$$
\begin{aligned}
M^{+}= & \left\{\mu \in M:\langle\mu, \psi\rangle_{\Gamma_{C}} \geq 0\right. \\
& \text { for all } \left.\psi \in N, \psi \geq 0 \text { a.e. on } \Gamma_{C}\right\},
\end{aligned}
$$

where the notation $\langle\cdot, \cdot\rangle_{\Gamma_{C}}$ represents the duality pairing between $M$ and $N$. Define

$$
\begin{aligned}
a(\boldsymbol{u}, \boldsymbol{v}) & =\int_{\Omega} \boldsymbol{\sigma}(\boldsymbol{u}): \boldsymbol{\varepsilon}(\boldsymbol{v}) \mathrm{d} \Omega, \quad b(\mu, \boldsymbol{v})=\left\langle\mu, v_{n}\right\rangle_{\Gamma_{C}}, \\
L(\boldsymbol{v}) & =\int_{\Omega} \boldsymbol{f} \cdot \boldsymbol{v} d \Omega+\int_{\Gamma_{N}} \boldsymbol{g} \cdot \boldsymbol{v} \mathrm{d} \Gamma
\end{aligned}
$$

for any $\boldsymbol{u}$ and $\boldsymbol{v}$ in $\boldsymbol{V}$ and $\mu$ in $M$. 
The mixed formulation of the unilateral contact problem without friction (1)-(6) consists then in finding $u \in$ $\boldsymbol{V}$ and $\lambda \in M^{+}$such that

$$
\left\{\begin{array}{l}
a(\boldsymbol{u}, \boldsymbol{v})+b(\lambda, \boldsymbol{v})=L(\boldsymbol{v}), \quad \forall \boldsymbol{v} \in \boldsymbol{V}, \\
b(\mu-\lambda, \boldsymbol{u}) \leq 0, \quad \forall \mu \in M^{+} .
\end{array}\right.
$$

An equivalent formulation of (7) consists in finding $(\lambda, \boldsymbol{u}) \in M^{+} \times \boldsymbol{V}$ satisfying

$\mathcal{L}(\mu, \boldsymbol{u}) \leq \mathcal{L}(\lambda, \boldsymbol{u}) \leq \mathcal{L}(\lambda, \boldsymbol{v}), \quad \forall \boldsymbol{v} \in \boldsymbol{V}, \forall \mu \in M^{+}$, where $\mathcal{L}(\mu, \boldsymbol{v})=\frac{1}{2} a(\boldsymbol{v}, \boldsymbol{v})-L(\boldsymbol{v})+b(\mu, \boldsymbol{v})$. Another classical weak formulation of the problem (1)- (6) is a variational inequality: Find $\boldsymbol{u}$ such that

$$
\boldsymbol{u} \in \boldsymbol{K}, \quad a(\boldsymbol{u}, \boldsymbol{v}-\boldsymbol{u}) \geq L(\boldsymbol{v}-\boldsymbol{u}), \quad \forall \boldsymbol{v} \in \boldsymbol{K}
$$

where $\boldsymbol{K}$ denotes the closed convex cone of admissible displacement fields satisfying the non-penetration conditions:

$$
\boldsymbol{K}=\left\{\boldsymbol{v} \in \boldsymbol{V}: \quad v_{n} \leq 0 \text { on } \Gamma_{C}\right\} .
$$

The existence and uniqueness of the solution $(\lambda, \boldsymbol{u})$ to (7) was given by Haslinger et al. (1996). Moreover, the first argument $\boldsymbol{u}$ solution to (7) is also the unique solution of the problem $(8)$ and $\lambda=-\sigma_{n}(\boldsymbol{u})$.

\section{Finite element approximation}

A regular family of triangulations denoted by $T_{h}$ is associated with the body $\Omega$ (see Brenner and Scott, 2002; Ciarlet, 1991). The closed triangles $K \in T_{h}$ are of diameter $h_{K}$, and we set $h=\max _{K \in T_{h}} h_{K}$. In order to use inverse inequalities on the contact area, we suppose that the one-dimensional mesh inherited on $\Gamma_{C}$ is uniformly regular, and we denote by $h_{C}$ a parameter representing the size of the elements on the contact zone (if the entire mesh is uniformly regular, as will be the case in the computations, we can merely choose $h_{C}=h$ ).

The finite dimensional space involving continuous affine finite elements is

$$
\begin{aligned}
\boldsymbol{V}_{h}=\left\{\boldsymbol{v}_{h} \in(C(\bar{\Omega}))^{2}: \forall \kappa \in T_{h}, \boldsymbol{v}_{h_{\left.\right|_{\kappa}}} \in\left(P_{1}(\kappa)\right)^{2},\right. \\
\left.\boldsymbol{v}_{h_{\left.\right|_{\Gamma_{D}}}}=\mathbf{0}\right\} .
\end{aligned}
$$

The normal trace space on the contact zone is defined as

$$
\begin{array}{r}
W_{h}=\left\{\mu_{h} \in C\left(\overline{\Gamma_{C}}\right): \exists \boldsymbol{v}_{h} \in \boldsymbol{V}_{h}\right. \\
\text { s.t. } \left.\boldsymbol{v}_{h} \cdot \boldsymbol{n}=\mu_{h} \text { on } \Gamma_{C}\right\},
\end{array}
$$

and the nonnegative functions of $W_{h}$ become

$$
W_{h}^{+}=\left\{\mu_{h} \in W_{h}: \mu_{h} \geq 0\right\} \text {. }
$$

The discrete problem approximating (7) is the following: Find $\boldsymbol{u}_{h} \in \boldsymbol{V}_{h}$ and $\lambda_{h} \in W_{h}^{+}$such that

$$
\begin{cases}a\left(\boldsymbol{u}_{h}, \boldsymbol{v}_{h}\right)+\int_{\Gamma_{C}} I_{h}\left(\lambda_{h} v_{h n}\right) \mathrm{d} \Gamma= & L\left(\boldsymbol{v}_{h}\right), \\ & \forall \boldsymbol{v}_{h} \in \boldsymbol{V}_{h}, \\ \int_{\Gamma_{C}} I_{h}\left(\left(\mu_{h}-\lambda_{h}\right) u_{h n}\right) \mathrm{d} \Gamma \leq 0, & \forall \mu_{h} \in W_{h}^{+},\end{cases}
$$

where $I_{h}$ stands for the standard Lagrange interpolation operator of degree 1 defined at the nodes of $\bar{\Gamma}_{C}: \forall v \in$ $C\left(\Gamma_{C}\right): I_{h} v \in C\left(\Gamma_{C}\right), I_{h} v(\boldsymbol{x})=v(\boldsymbol{x})$ for any node $\boldsymbol{x}$ in $\bar{\Gamma}_{C}$, and $I_{h} v$ is an affine function between two nodes. The following proposition proves the existence of a unique solution to the problem (9). It also gives some elementary properties of the solution and describes links with a standard variational inequality.

\section{Proposition 1.}

(i) The problem (9) admits a unique solution $\left(\lambda_{h}, \boldsymbol{u}_{h}\right) \in$ $W_{h}^{+} \times \boldsymbol{V}_{h}$.

(ii) One has $u_{h n} \leq 0, \lambda_{h} \geq 0$ on $\Gamma_{C}$ and $\lambda_{h} u_{h n}=$ 0 at the nodes of $\Gamma_{C}$.

(iii) The displacement field $\boldsymbol{u}_{h}$ solving (9) is the unique solution to the problem: Find $\boldsymbol{u}_{h} \in \boldsymbol{K}_{h}=$ $\left\{\boldsymbol{v}_{h} \in \boldsymbol{V}_{h}: v_{h n} \leq 0\right.$ on $\left.\Gamma_{C}\right\}$ such that

$$
a\left(\boldsymbol{u}_{h}, \boldsymbol{v}_{h}-\boldsymbol{u}_{h}\right) \geq L\left(\boldsymbol{v}_{h}-\boldsymbol{u}_{h}\right), \quad \forall \boldsymbol{v}_{h} \in \boldsymbol{K}_{h} .
$$

Proof.

(i) Since we deal with the finite dimensional case, we only need to check (see Theorem 3.9 and Example 3.8 of Haslinger et al., 1996) that

$$
\sup _{\boldsymbol{v}_{h} \in \boldsymbol{V}_{h}, \boldsymbol{v}_{h} \neq \mathbf{0}} \frac{\int_{\Gamma_{C}} I_{h}\left(\mu_{h} v_{h n}\right) \mathrm{d} \Gamma}{\left\|\boldsymbol{v}_{h}\right\|_{1, \Omega}}
$$

is a norm on $W_{h}$. Thus we have to verify that

$$
\left\{\mu_{h} \in W_{h}: \int_{\Gamma_{C}} I_{h}\left(\mu_{h} v_{h n}\right) \mathrm{d} \Gamma=0, \forall \boldsymbol{v}_{h} \in \boldsymbol{V}_{h}\right\}=\{0\},
$$

which is satisfied according to the definition of $W_{h}$. Hence the problem (9) admits a unique solution $\left(\lambda_{h}, \boldsymbol{u}_{h}\right) \in W_{h}^{+} \times \boldsymbol{V}_{h}$

(ii) Set

$$
\begin{aligned}
& c\left(\mu_{h}, \boldsymbol{v}_{h}\right)=\int_{\Gamma_{C}} I_{h}\left(\mu_{h} v_{h n}\right) \mathrm{d} \Gamma, \quad \forall \mu_{h} \in W_{h}, \\
& \forall \boldsymbol{v}_{h} \in \boldsymbol{V}_{h} .
\end{aligned}
$$


Taking $\mu_{h}=0$ and $\mu_{h}=2 \lambda_{h}$ in (9) leads to

$$
c\left(\lambda_{h}, \boldsymbol{u}_{h}\right)=0 \quad \text { and } \quad c\left(\mu_{h}, \boldsymbol{u}_{h}\right) \leq 0, \quad \forall \mu_{h} \in W_{h}^{+} .
$$

Taking $\mu_{h}=\psi_{x} \in W_{h}^{+}$in the previous inequality where $\psi_{x}$ is the scalar basis function of $W_{h}$ (defined on $\bar{\Gamma}_{C}$ ) at node $x \in \bar{\Gamma}_{C}$ satisfying $\psi_{x}\left(x^{\prime}\right)=\delta_{x, x^{\prime}}$ for any node $x^{\prime} \in$ $\bar{\Gamma}_{C}$, we deduce that $u_{h n}(x) \leq 0$. Hence $u_{h n} \leq 0$ on $\Gamma_{C}$.

From $\lambda_{h} u_{h n} \leq 0$ on $\Gamma_{C}$ and since $c\left(\lambda_{h}, \boldsymbol{u}_{h}\right)=0$, we come to the conclusion that $I_{h}\left(\lambda_{h} u_{h n}\right)=0$ on $\Gamma_{C}$. That proves point (ii).

(iii) From (9) and $c\left(\lambda_{h}, \boldsymbol{u}_{h}\right)=0$, we get

$$
a\left(\boldsymbol{u}_{h}, \boldsymbol{u}_{h}\right)=L\left(\boldsymbol{u}_{h}\right)
$$

and for any $\boldsymbol{v}_{h} \in \boldsymbol{K}_{h}$, we obtain

$$
a\left(\boldsymbol{u}_{h}, \boldsymbol{v}_{h}\right)-L\left(\boldsymbol{v}_{h}\right)=-\int_{\Gamma_{C}} I_{h}\left(\lambda_{h} v_{h n}\right) \mathrm{d} \Gamma \geq 0 .
$$

Putting together (11) and (12) implies that $\boldsymbol{u}_{h}$ is a solution of the variational inequality (10) which admits a unique solution according to Stampacchia's theorem.

The standard approach (see, e.g., Ben Belgacem and Renard, 2003; Coorevits et al., 2002; Hüeber and Wohlmuth, 2005a) consists in solving the following discrete problem (using the same arguments as in the previous proposition, it admits a unique solution): Find $\boldsymbol{w}_{h} \in$ $\boldsymbol{V}_{h}$ and $\theta_{h} \in M_{h}^{+}$such that

$$
\left\{\begin{array}{l}
a\left(\boldsymbol{w}_{h}, \boldsymbol{v}_{h}\right)+b\left(\theta_{h}, \boldsymbol{v}_{h}\right)=L\left(\boldsymbol{v}_{h}\right), \quad \forall \boldsymbol{v}_{h} \in \boldsymbol{V}_{h}, \\
b\left(\mu_{h}-\theta_{h}, \boldsymbol{w}_{h}\right) \leq 0, \quad \forall \mu_{h} \in M_{h}^{+},
\end{array}\right.
$$

where

$$
M_{h}^{+}=\left\{\mu_{h} \in W_{h}: \int_{\Gamma_{C}} \mu_{h} \psi_{h} \mathrm{~d} \Gamma \geq 0, \forall \psi_{h} \in W_{h}^{+}\right\} .
$$

Remark 1. We have $W_{h}^{+} \subset M^{+}$and $W_{h}^{+} \subset M_{h}^{+} \not \subset$ $M^{+}$.

The next proposition establishes a link between the solutions of the problems (9) and (13).

Proposition 2. The solutions $\left(\lambda_{h}, \boldsymbol{u}_{h}\right)$ and $\left(\theta_{h}, \boldsymbol{w}_{h}\right)$ of the problems (9) and (13) satisfy what follows:

(i) $\boldsymbol{u}_{h}=\boldsymbol{w}_{h}$,

(ii) $\lambda_{h}=\pi_{h} \theta_{h}$, where $\pi_{h}: L^{1}\left(\Gamma_{C}\right) \mapsto W_{h}$ is the quasi-interpolation operator defined for any function $v$ in $L^{1}\left(\Gamma_{C}\right)$ by

$$
\pi_{h} v=\sum_{x \in N_{h}} \alpha_{x}(v) \psi_{x}
$$

$N_{h}$ represents the set of nodes of $\bar{\Gamma}_{C}, \psi_{x}$ is the scalar basis function of $W_{h}$ (defined on $\bar{\Gamma}_{C}$ ) at the node $x$ satisfying $\psi_{x}\left(x^{\prime}\right)=\delta_{x, x^{\prime}}$ for all $x^{\prime} \in N_{h}$ and

$$
\alpha_{x}(v)=\left(\int_{\Gamma_{C}} v \psi_{x} \mathrm{~d} \Gamma\right)\left(\int_{\Gamma_{C}} \psi_{x} \mathrm{~d} \Gamma\right)^{-1} .
$$

Proof.

(i) The same discussion as in points (ii) and (iii) of Proposition 1 and some polarity arguments (see, e.g., Hild, 2000; Hild and Nicaise, 2007) which we describe hereafter prove that $\boldsymbol{w}_{h}$ is also the unique solution of the variational inequality (10). Let us briefly summarize the result: Choosing $\mu_{h}=0$ and $\mu_{h}=2 \theta_{h}$ in 13 implies $b\left(\theta_{h}, \boldsymbol{w}_{h}\right)=0$ and $b\left(\mu_{h}, \boldsymbol{w}_{h}\right)=\int_{\Gamma_{C}} \mu_{h} w_{h n} \mathrm{~d} \Gamma \leq$ $0, \forall \mu_{h} \in M_{h}^{+}$. Consequently, $w_{h n} \in-\left(M_{h}^{+}\right)^{*}$ (the notation $X^{*}$ stands for the positive polar cone of $X$ for the inner product on $W_{h}$ induced by $b(\cdot, \cdot)$, (Hiriart-Urruty and Lemaréchal, 1993, p. 119). We have $\left(M_{h}^{+}\right)^{*}=$ $\left(\left(W_{h}^{+}\right)^{*}\right)^{*}=W_{h}^{+}$since $W_{h}^{+}$is a closed convex cone. Hence $w_{h n} \in-W_{h}^{+}$and $\boldsymbol{w}_{h} \in \boldsymbol{K}_{h}$. Besides, (13) and $b\left(\theta_{h}, \boldsymbol{w}_{h}\right)=0$ lead to $a\left(\boldsymbol{w}_{h}, \boldsymbol{w}_{h}\right)=L\left(\boldsymbol{w}_{h}\right)$, and for any $\boldsymbol{v}_{h} \in \boldsymbol{K}_{h}$, we get

$$
a\left(\boldsymbol{w}_{h}, \boldsymbol{v}_{h}\right)-L\left(\boldsymbol{v}_{h}\right)=-\int_{\Gamma_{C}} \theta_{h} v_{h n} \mathrm{~d} \Gamma \geq 0
$$

since $\theta_{h} \in M_{h}^{+}=\left(W_{h}^{+}\right)^{*}$ and $v_{h n} \in-W_{h}^{+}$. Hence $\boldsymbol{w}_{h}$ is the unique solution of the variational inequality (10) and point (iii) of Proposition 1 establishes the result.

(ii) From (i) and the equalities in (9) and (13), we deduce that

$$
\int_{\Gamma_{C}} \theta_{h} v_{h n} \mathrm{~d} \Gamma=\int_{\Gamma_{C}} I_{h}\left(\lambda_{h} v_{h n}\right) \mathrm{d} \Gamma, \quad \forall \boldsymbol{v}_{h} \in \boldsymbol{V}_{h} .
$$

We choose $\boldsymbol{v}_{h}$ such that $v_{h n}=\psi_{x}$ where $\psi_{x}$ is the scalar basis function of $W_{h}$ at node $x \in \bar{\Gamma}_{C}$. As a consequence,

$$
\int_{\Gamma_{C}} \theta_{h} \psi_{x} \mathrm{~d} \Gamma=\lambda_{h}(x) \int_{\Gamma_{C}} \psi_{x} \mathrm{~d} \Gamma
$$

This proves that $\lambda_{h}=\pi_{h} \theta_{h}$, where $\pi_{h}$ is the linear operator defined above.

\section{Positivity preserving averaging operator: Basic properties}

Now, we intend to study the basic properties of the operator $\pi_{h}$ defined in Proposition 2. It is obvious that $\pi_{h}$ is a linear averaging operator (for other averaging operators, see, e.g., Bernardi and Girault, 1998; Chen and Nochetto, 2000; Clément, 1975; Hilbert, 1973; Scott and Zhang, 1990; Strang, 1972), and that it not only preserves the nonnegative functions, but also satisfies $\pi_{h}\left(M_{h}^{+}\right)=$ $W_{h}^{+}$, which means that it transforms finite element type functions with a weak nonnegativity condition into nonnegative functions (such a property is also satisfied by the operator in the work of Chen and Nochetto (2000)). For a detailed discussion concerning positivity preserving finite element approximation, we refer the reader to the work of Nochetto and Wahlbin (2002). Obviously, $\pi_{h} v_{h} \neq v_{h}$ in the general case when $v_{h} \in W_{h}$. Moreover, it is easy to 
see that $\pi_{h}\left(W_{h}\right)=W_{h}$. Finally, it is straightforward to check that any locally constant function is reproduced locally by $\pi_{h}$ (this is not the case for locally affine functions, since the meshes on $\Gamma_{C}$ do not have the same length), and that

$$
\int_{\Gamma_{C}} v-\pi_{h} v \mathrm{~d} \Gamma=0
$$

for any $v \in L^{1}\left(\Gamma_{C}\right)$, which means that the operator preserves globally the average (note that a local average preserving property does not hold). In the following proofs, we denote by $C$ a positive generic constant independent of the discretization parameter $h$. Now we show the $L^{2}$ stability property of $\pi_{h}$.

Lemma 1. There is a positive constant $C$ independent of $h$ such that for any $v \in L^{2}\left(\Gamma_{C}\right)$ and any $E \in E_{h}^{C}\left(E_{h}^{C}\right.$ denotes the set of closed edges lying in $\bar{\Gamma}_{C}$ )

$$
\left\|\pi_{h} v\right\|_{0, E} \leq C\|v\|_{0, \gamma_{E}}
$$

where $\gamma_{E}=\cup_{\left\{F \in E_{h}^{C}: F \cap E \neq \emptyset\right\}} F$.

Proof. Let $\gamma_{x}$ be the support of the basis function $\psi_{x}$ in $\Gamma_{C}$. Using the definition of $\alpha_{x}(v)$ in Proposition 2, the Cauchy-Schwarz inequality, and the uniform regularity, we get

$$
\begin{aligned}
\left|\alpha_{x}(v)\right| & \leq\|v\|_{0, \gamma_{x}}\left\|\psi_{x}\right\|_{0, \gamma_{x}}\left\|\psi_{x}\right\|_{L^{1}\left(\gamma_{x}\right)}^{-1} \\
& \leq C h_{C}^{-\frac{1}{2}}\|v\|_{0, \gamma_{x}} .
\end{aligned}
$$

Denoting by $N_{h}$ the set of nodes of $\bar{\Gamma}_{C}$, we obtain by a triangular inequality

$$
\left\|\pi_{h} v\right\|_{0, E}=\left\|\sum_{x \in N_{h} \cap E} \alpha_{x}(v) \psi_{x}\right\|_{0, E} \leq C\|v\|_{0, \gamma_{E}} .
$$

The next lemma is concerned with the $L^{2}$ approximation properties of $\pi_{h}$.

Lemma 2. There is a positive constant $C$ independent of $h$ such that for any $v \in H^{\eta}\left(\Gamma_{C}\right), 0 \leq \eta \leq 1$, and any $E \in E_{h}^{C}$ ( $E_{h}^{C}$ denotes the set of closed edges lying in $\bar{\Gamma}_{C}$ )

$$
\left\|v-\pi_{h} v\right\|_{0, E} \leq C h^{\eta}\|v\|_{\eta, \gamma_{E}}
$$

where $\gamma_{E}=\cup_{\left\{F \in E_{h}^{C}: F \cap E \neq \emptyset\right\}} F$.

Proof. When $\eta=0$, the bound results from the previous lemma. Note that $\pi_{h}$ preserves the constant functions on $\Gamma_{C}$. Let there be given an arbitrary constant function $c(x)=c, \forall x \in \Gamma_{C}$. From the definition of $\pi_{h}$, we may write for any $v \in H^{\eta}\left(\Gamma_{C}\right)$

$$
v-\pi_{h} v=v-c-\pi_{h}(v-c) .
$$

Therefore, by Lemma 1 we get

$$
\begin{aligned}
\left\|v-\pi_{h} v\right\|_{0, E} & \leq C\left(\|v-c\|_{0, E}+\|v-c\|_{0, \gamma_{E}}\right) \\
& \leq C\|v-c\|_{0, \gamma_{E}}, \quad \forall c \in \mathbb{R} .
\end{aligned}
$$

We then choose $c=\int_{\gamma_{E}} v(x) \mathrm{d} x /\left|\gamma_{E}\right|$ in (17), where $\left|\gamma_{E}\right|$ denotes the length of $\gamma_{E}$. Then, if $x \in \gamma_{E}$ and $0<\eta<1$, we have

$$
\begin{aligned}
v(x)-c & =\left|\gamma_{E}\right|^{-1} \int_{\gamma_{E}} v(x)-v(y) \mathrm{d} y \\
& =\left|\gamma_{E}\right|^{-1} \int_{\gamma_{E}} \frac{v(x)-v(y)}{|x-y|^{\frac{1+2 \eta}{2}}}|x-y|^{\frac{1+2 \eta}{2}} \mathrm{~d} y .
\end{aligned}
$$

Using the Cauchy-Schwarz inequality, we deduce that

$$
\begin{aligned}
& \int_{\gamma_{E}}(v(x)-c)^{2} \mathrm{~d} x \\
& =\left|\gamma_{E}\right|^{-2} \int_{\gamma_{E}}\left(\int_{\gamma_{E}} \frac{v(x)-v(y)}{|x-y|^{\frac{1+2 \eta}{2}}}|x-y|^{\frac{1+2 \eta}{2}} \mathrm{~d} y\right)^{2} \mathrm{~d} x \\
& \leq\left|\gamma_{E}\right|^{-2} \\
& \times \int_{\gamma_{E}}\left(\int_{\gamma_{E}} \frac{(v(x)-v(y))^{2}}{|x-y|^{1+2 \eta}} \mathrm{d} y \int_{\gamma_{E}}|x-y|^{1+2 \eta} \mathrm{d} y\right) \mathrm{d} x \\
& \leq\left|\gamma_{E}\right|^{2 \eta} \int_{\gamma_{E}} \int_{\gamma_{E}} \frac{(v(x)-v(y))^{2}}{|x-y|^{1+2 \eta}} \mathrm{d} y \mathrm{~d} x \\
& \leq C h^{2 \eta}\|v\|_{\eta, \gamma_{E}}^{2},
\end{aligned}
$$

which is our claim.

If $x \in \gamma_{E}$ and $\eta=1$, we have

$$
\begin{aligned}
v(x)-c & =\left|\gamma_{E}\right|^{-1} \int_{\gamma_{E}} v(x)-v(y) \mathrm{d} y \\
& =\left|\gamma_{E}\right|^{-1} \int_{\gamma_{E}} \int_{y}^{x} v^{\prime}(t) \mathrm{d} t \mathrm{~d} y,
\end{aligned}
$$

where the notation $v^{\prime}$ stands for the derivative of $v$. Hence

$$
|v(x)-c| \leq\left|\gamma_{E}\right|^{\frac{1}{2}}\left\|v^{\prime}\right\|_{0, \gamma_{E}} .
$$

The result is then straightforward.

An open question is concerned with the optimal approximation properties of $\pi_{h}$ in dual Sobolev spaces (typically $\left.H^{-\frac{1}{2}}\left(\Gamma_{C}\right)\right)$. It is easily seen that the $L^{2}\left(\Gamma_{C}\right)$ projection operator onto continuous and piecewise affine functions as well as the $L^{2}\left(\Gamma_{C}\right)$-projection operator onto piecewise constant functions satisfy such properties. On the contrary, it can be shown that the Lagrange interpolation operator as well as the $L^{2}\left(\Gamma_{C}\right)$-projection operator applied to nonnegative functions and mapping onto $W_{h}^{+}$ do not fulfil such properties. Unfortunately, the counter examples for the last two operators use the fact that the average of the function is not preserved and this is not the case for $\pi_{h}$ (see (16). 


\section{A priori error estimates}

Now we intend to analyze the convergence of the finite element problem (9). In the forthcoming error analysis we suppose that $\boldsymbol{u} \in\left(H^{\frac{3}{2}+\eta}(\Omega)\right)^{2}$ with $0<\eta \leq 1 / 2$, which implies that $u_{n}$ is continuous on $\Gamma_{C}$ (which is a straight line segment). Set

$$
\begin{aligned}
& \gamma_{c}=\left\{x \in \Gamma_{C}: u_{n}(x)=0\right\}, \\
& \gamma_{s}=\Gamma_{C} \backslash \gamma_{c} .
\end{aligned}
$$

In order to obtain an optimal convergence rate, we have to use the following assumption:

$$
\text { The number of points in } \overline{\gamma_{c}} \cap \overline{\gamma_{s}} \text { is finite. }
$$

The case where (18) is not valid is considered by Corollary 1 Let us first recall the result established in Hüeber and Wohlmuth (2005a).

Lemma 3. (Hüeber and Wohlmuth, 2005) Let $(\lambda, \boldsymbol{u})$ be the solution of (7), and let $\left(\theta_{h}, \boldsymbol{u}_{h}\right)$ be the solution of (13). Assume that (18) holds. Let the regularity assumption $\boldsymbol{u} \in$ $\left(H^{\frac{3}{2}+\eta}(\Omega)\right)^{2}$ with $0<\eta \leq 1 / 2$ hold. Then there exists a positive constant $C$ independent of $h$ and satisfying

$$
\left\|\boldsymbol{u}-\boldsymbol{u}_{h}\right\|_{1, \Omega}+\left\|\lambda-\theta_{h}\right\|_{-\frac{1}{2}, \Gamma_{C}} \leq C h^{\frac{1}{2}+\eta}\|\boldsymbol{u}\|_{\frac{3}{2}+\eta, \Omega} .
$$

This result and the triangle inequality imply the bound in the next lemma.

Lemma 4. Let $(\lambda, \boldsymbol{u})$ be the solution of (7), let $\left(\lambda_{h}, \boldsymbol{u}_{h}\right)$ be the solution of (9), and let $\left(\theta_{h}, \boldsymbol{u}_{h}\right)$ be the solution of (13). Assume that (18) holds. Let the regularity assumption $\boldsymbol{u} \in\left(H^{\frac{3}{2}+\eta}(\Omega)\right)^{2}$ with $0<\eta \leq 1 / 2$ hold. Then there exists a positive constant $C$ independent of $h$ satisfying

$$
\begin{aligned}
\left\|\boldsymbol{u}-\boldsymbol{u}_{h}\right\|_{1, \Omega}+\left\|\lambda-\lambda_{h}\right\|_{-\frac{1}{2}, \Gamma_{C}} \\
\quad \leq C h^{\frac{1}{2}+\eta}\|\boldsymbol{u}\|_{\frac{3}{2}+\eta, \Omega}+\left\|\lambda_{h}-\theta_{h}\right\|_{-\frac{1}{2}, \Gamma_{C}} .
\end{aligned}
$$

Now we have to estimate the term $\left\|\lambda_{h}-\theta_{h}\right\|_{-\frac{1}{2}, \Gamma_{C}}$. A first bound is given hereafter.

Lemma 5. Assume that the hypotheses of Lemma 4 hold. Then there exists a positive constant $C$ independent of $h$ and satisfying

$$
\begin{aligned}
\| \lambda_{h}- & \theta_{h} \|_{-\frac{1}{2}, \Gamma_{C}} \\
& \leq C\left(h^{\frac{1}{2}+\eta}\|\boldsymbol{u}\|_{\frac{3}{2}+\eta, \Omega}+h_{C}^{\frac{1}{2}}\left\|\lambda_{h}-\theta_{h}\right\|_{0, \Gamma_{C}}\right) .
\end{aligned}
$$

Proof. From the discrete inf-sup condition (see, e.g., Coorevits et al., 2002)

$$
0<C \leq \inf _{\mu_{h} \in W_{h}} \sup _{\boldsymbol{v}_{h} \in \boldsymbol{V}_{h}} \frac{b\left(\mu_{h}, \boldsymbol{v}_{h}\right)}{\left\|\mu_{h}\right\|_{-\frac{1}{2}, \Gamma_{C}}\left\|\boldsymbol{v}_{h}\right\|_{1, \Omega}}
$$

and 15 , we get

$$
\begin{aligned}
& \left\|\lambda_{h}-\theta_{h}\right\|_{-\frac{1}{2}, \Gamma_{C}} \\
& \leq C \sup _{\boldsymbol{v}_{h} \in \boldsymbol{V}_{h}} \frac{b\left(\lambda_{h}-\theta_{h}, \boldsymbol{v}_{h}\right)}{\left\|\boldsymbol{v}_{h}\right\|_{1, \Omega}} \\
& =C \sup _{\boldsymbol{v}_{h} \in \boldsymbol{V}_{h}} \frac{\int_{\Gamma_{C}} \lambda_{h} v_{h n}-I_{h}\left(\lambda_{h} v_{h n}\right) \mathrm{d} \Gamma}{\left\|\boldsymbol{v}_{h}\right\|_{1, \Omega}} .
\end{aligned}
$$

Besides, we have

$$
\begin{aligned}
\int_{\Gamma_{C}} \lambda_{h} v_{h n}- & I_{h}\left(\lambda_{h} v_{h n}\right) \mathrm{d} \Gamma \\
& =\sum_{E \in E_{h}^{C}} \int_{E} \lambda_{h} v_{h n}-I_{h}\left(\lambda_{h} v_{h n}\right) \mathrm{d} \Gamma
\end{aligned}
$$

where $E_{\underline{h}}^{C}$ denotes the set of closed edges (of triangles) lying in $\bar{\Gamma}_{C}$. From numerical integration (trapezoidal formula) and the Cauchy-Schwarz inequality, we get

$$
\begin{aligned}
\int_{E} \lambda_{h} v_{h n}-I_{h}\left(\lambda_{h} v_{h n}\right) \mathrm{d} \Gamma \\
\quad \leq C h_{E}^{3}\left|\left(\lambda_{h} v_{h n}\right)_{\left.\right|_{E}}^{\prime \prime}\right| \\
\leq C h_{E}^{3}\left|\left(\lambda_{h}^{\prime} v_{h n}^{\prime}\right)\right|_{E} \mid \\
\leq C h_{E}^{2}\left\|\lambda_{h}^{\prime}\right\|_{0, E}\left\|v_{h n}^{\prime}\right\|_{0, E} \\
\quad=C h_{E}^{2}\left\|\left(\lambda_{h}-\bar{\lambda}\right)^{\prime}\right\|_{0, E}\left\|v_{h n}^{\prime}\right\|_{0, E}
\end{aligned}
$$

where $h_{E}$ denotes the length of the edge $E, \bar{\lambda}=$ $\left(\int_{E} \lambda d \Gamma\right) / h_{E}$, and $v^{\prime}, v^{\prime \prime}$ denote the derivatives of the first and second orders of $v$. An inverse inequality implies

$$
\begin{aligned}
& \int_{E} \lambda_{h} v_{h n}-I_{h}\left(\lambda_{h} v_{h n}\right) \mathrm{d} \Gamma \\
& \leq C h_{E}\left\|\lambda_{h}-\bar{\lambda}\right\|_{0, E}\left\|v_{h n}^{\prime}\right\|_{0, E} .
\end{aligned}
$$

Writing $\boldsymbol{v}_{h}=\left(v_{h x}, v_{h y}\right)$, we can suppose without loss of generality that $\Gamma_{C}$ is parallel to the horizontal $x$ axis (the $y$-axis is vertical). Using the scaled trace theorem (see, e.g., Grisvard, 1985),

$$
\begin{array}{r}
\|v\|_{0, E} \leq C\left(h_{E}^{-\frac{1}{2}}\|v\|_{0, K}+h_{E}^{\frac{1}{2}}\|\nabla v\|_{0, K}\right), \\
\forall E \in E_{K}, \forall v \in H^{1}(K),
\end{array}
$$

( $E_{K}$ represents the set of the three edges belonging to the triangle $K$ ), we deduce that

$$
\begin{aligned}
\left\|v_{h n}^{\prime}\right\|_{0, E} & =\left\|\frac{\partial v_{h y}}{\partial x}\right\|_{0, E} \\
& \leq C h_{E}^{-\frac{1}{2}}\left\|\frac{\partial v_{h y}}{\partial x}\right\|_{0, K} \\
& \leq C h_{E}^{-\frac{1}{2}}\left\|v_{h y}\right\|_{1, K} \leq C h_{E}^{-\frac{1}{2}}\left\|\boldsymbol{v}_{h}\right\|_{1, K} .
\end{aligned}
$$


Hence

$$
\begin{aligned}
& \int_{E} \lambda_{h} v_{h n}-I_{h}\left(\lambda_{h} v_{h n}\right) \mathrm{d} \Gamma \\
& \leq C h_{E}^{\frac{1}{2}}\left\|\lambda_{h}-\bar{\lambda}\right\|_{0, E}\left\|\boldsymbol{v}_{h}\right\|_{1, K} .
\end{aligned}
$$

Therefore, denoting again by $\bar{\lambda}$ the piecewise constant function defined on $\Gamma_{C}$ such that $\bar{\lambda}_{\left.\right|_{E}}=\left(\int_{E} \lambda \mathrm{d} \Gamma\right) / h_{E}$, we obtain by addition

$$
\begin{aligned}
& \int_{\Gamma_{C}} \lambda_{h} v_{h n}-I_{h}\left(\lambda_{h} v_{h n}\right) \mathrm{d} \Gamma \\
& \leq C h_{C}^{\frac{1}{2}}\left\|\bar{\lambda}-\lambda_{h}\right\|_{0, \Gamma_{C}}\left\|\boldsymbol{v}_{h}\right\|_{1, \Omega} .
\end{aligned}
$$

According to 19], we deduce that

$$
\begin{aligned}
\| \lambda_{h} & -\theta_{h} \|_{-\frac{1}{2}, \Gamma_{C}} \\
\leq & C h_{C}^{\frac{1}{2}}\left\|\bar{\lambda}-\lambda_{h}\right\|_{0, \Gamma_{C}} \\
\leq & C h_{C}^{\frac{1}{2}}\left(\left\|\lambda-\theta_{h}\right\|_{0, \Gamma_{C}}\right. \\
& \left.+\left\|\theta_{h}-\lambda_{h}\right\|_{0, \Gamma_{C}}+\|\lambda-\bar{\lambda}\|_{0, \Gamma_{C}}\right) .
\end{aligned}
$$

Then we use the standard estimate $\|\lambda-\bar{\lambda}\|_{0, \Gamma_{C}} \leq$ $C h^{\eta}\|\lambda\|_{\eta, \Gamma_{C}}$ (the latter result is obtained in the proof of Lemma 2) together with the trace theorem (the coefficients in the elasticity operator are supposed to lie in $C^{1}(\bar{\Omega})$ ).

The term $\left\|\lambda-\theta_{h}\right\|_{0, \Gamma_{C}}$ is estimated by using an inverse inequality, Lemma 3 , and the optimal approximation properties in $H^{-\frac{1}{2}}\left(\Gamma_{C}\right)$ of the $L^{2}\left(\Gamma_{C}\right)$-projection operator $p_{h}$ mapping onto $W_{h}$. We recall that $p_{h}$ is defined for any $v \in L^{2}\left(\Gamma_{C}\right)$ by

$$
p_{h} v \in W_{h}, \quad \int_{\Gamma_{C}}\left(v-p_{h} v\right) \psi_{h} \mathrm{~d} \Gamma=0, \quad \forall \psi_{h} \in W_{h} .
$$

More precisely, we have

$$
\begin{aligned}
\| \lambda- & \theta_{h} \|_{0, \Gamma_{C}} \\
\leq & \left\|\lambda-p_{h} \lambda\right\|_{0, \Gamma_{C}}+\left\|p_{h} \lambda-\theta_{h}\right\|_{0, \Gamma_{C}} \\
\leq & C\left(h^{\eta}\|\lambda\|_{\eta, \Gamma_{C}}+h_{C}^{-\frac{1}{2}}\left\|p_{h} \lambda-\theta_{h}\right\|_{-\frac{1}{2}, \Gamma_{C}}\right) \\
\leq & C\left(h^{\eta}\|\boldsymbol{u}\|_{\frac{3}{2}+\eta, \Omega}+h_{C}^{-\frac{1}{2}}\left\|p_{h} \lambda-\lambda\right\|_{-\frac{1}{2}, \Gamma_{C}}\right. \\
& \left.+h_{C}^{-\frac{1}{2}}\left\|\lambda-\theta_{h}\right\|_{-\frac{1}{2}, \Gamma_{C}}\right)
\end{aligned}
$$

and

$$
h_{C}^{\frac{1}{2}}\left\|\lambda-\theta_{h}\right\|_{0, \Gamma_{C}} \leq C h^{\frac{1}{2}+\eta}\|\boldsymbol{u}\|_{\frac{3}{2}+\eta, \Omega}
$$

Finally,

$$
\begin{aligned}
\| \lambda_{h}- & \theta_{h} \|_{-\frac{1}{2}, \Gamma_{C}} \\
& \leq C\left(h^{\frac{1}{2}+\eta}\|\boldsymbol{u}\|_{\frac{3}{2}+\eta, \Omega}+h_{C}^{\frac{1}{2}}\left\|\lambda_{h}-\theta_{h}\right\|_{0, \Gamma_{C}}\right) .
\end{aligned}
$$

Lemma 6. Assume that the hypotheses of Lemma 4 hold. Then there exists a positive constant $C$ independent of $h$ and satisfying

$$
h_{C}^{\frac{1}{2}}\left\|\lambda_{h}-\theta_{h}\right\|_{0, \Gamma_{C}} \leq C h^{\frac{1}{2}+\eta}\|\boldsymbol{u}\|_{\frac{3}{2}+\eta, \Omega} .
$$

Proof. We write

$$
\begin{aligned}
\left\|\lambda_{h}-\theta_{h}\right\|_{0, \Gamma_{C}}= & \left\|\theta_{h}-\pi_{h} \theta_{h}\right\|_{0, \Gamma_{C}} \\
\leq & \left\|\left(\theta_{h}-\lambda\right)-\pi_{h}\left(\theta_{h}-\lambda\right)\right\|_{0, \Gamma_{C}} \\
& +\left\|\lambda-\pi_{h} \lambda\right\|_{0, \Gamma_{C}} .
\end{aligned}
$$

Using Lemma2 when adding the local estimates gives

$$
\left\|\lambda_{h}-\theta_{h}\right\|_{0, \Gamma_{C}} \leq C\left(\left\|\lambda-\theta_{h}\right\|_{0, \Gamma_{C}}+h^{\eta}\|\lambda\|_{\eta, \Gamma_{C}}\right),
$$

and the bound 21 yields

$$
h_{C}^{\frac{1}{2}}\left\|\lambda_{h}-\theta_{h}\right\|_{0, \Gamma_{C}} \leq C h^{\frac{1}{2}+\eta}\|\boldsymbol{u}\|_{\frac{3}{2}+\eta, \Omega} .
$$

We finally obtain the optimal a priori error estimate for the sign preserving method.

Theorem 1. Let $(\lambda, \boldsymbol{u})$ be the solution of (7) and let $\left(\lambda_{h}, \boldsymbol{u}_{h}\right)$ be the solution of (9). Assume that (18) holds. Let the regularity assumption $\boldsymbol{u} \in\left(H^{\frac{3}{2}+\eta}(\Omega)\right)^{2}$ with $0<$ $\eta \leq 1 / 2$ hold. Then, there exists a positive constant $C$ independent of $h$ satisfying

$$
\left\|\boldsymbol{u}-\boldsymbol{u}_{h}\right\|_{1, \Omega}+\left\|\lambda-\lambda_{h}\right\|_{-\frac{1}{2}, \Gamma_{C}} \leq C h^{\frac{1}{2}+\eta}\|\boldsymbol{u}\|_{\frac{3}{2}+\eta, \Omega} .
$$

Proof. It suffices to put together the results of Lemmas 4,6

Remark 2. If the operator $\pi_{h}$ satisfied optimal approximation properties in dual Sobolev spaces (as $H^{-\frac{1}{2}}\left(\Gamma_{C}\right)$ ), then the proof of Theorem 1 would be straightforward (in this case one could avoid Lemma 5) since it suffices to write $\left\|\lambda_{h}-\theta_{h}\right\|_{-\frac{1}{2}, \Gamma_{C}}=\left\|\theta_{h}-\pi_{h} \theta_{h}\right\|_{-\frac{1}{2}, \Gamma_{C}} \leq$ $\left\|\left(\lambda-\theta_{h}\right)-\pi_{h}\left(\lambda-\theta_{h}\right)\right\|_{-\frac{1}{2}, \Gamma_{C}}^{-\frac{1}{2}, \Gamma_{C}}+\left\|\lambda-\pi_{h} \lambda\right\|_{-\frac{1}{2}, \Gamma_{C}}$, and these properties (together with some inverse estimates) would end the proof. Unfortunately, such properties are not available (see also the discussion at the end of Section 4).

Remark 3. A deeper insight into the estimates shows that the direct error analysis of the finite element method (9) by circumventing the standard approximation (13) would be nontrivial (at least not shorter than the present analysis).

The assumption (18) is concerned with the finite number of transition points between contact and separation zones. Actually, we cannot prove that such an assumption is satisfied in practice. Without this hypothesis we can obtain a convergence result for the finite element method (9). This is achieved in the next corollary. 
Corollary 1. Let $(\lambda, \boldsymbol{u})$ be the solution of (7), and let $\left(\lambda_{h}, \boldsymbol{u}_{h}\right)$ be the solution of (9). Assume that $\boldsymbol{u} \in$ $\left(H^{\frac{3}{2}+\eta}(\Omega)\right)^{2}$ with $0<\eta \leq 1 / 2$. Then there exists a positive constant $C$ independent of $h$ and satisfying

$$
\left\|\boldsymbol{u}-\boldsymbol{u}_{h}\right\|_{1, \Omega}+\left\|\lambda-\lambda_{h}\right\|_{-\frac{1}{2}, \Gamma_{C}} \leq C h^{\frac{1+\eta}{2}}\|\boldsymbol{u}\|_{\frac{3}{2}+\eta, \Omega} .
$$

Proof. The result is straightforward by noting that the solution $\left(\theta_{h}, \boldsymbol{u}_{h}\right)$ of (13) satisfies, under the $\left(H^{\frac{3}{2}+\eta}(\Omega)\right)^{2}$ regularity hypothesis (see, e.g., Ben Belgacem et al., 1999)

$$
\left\|\boldsymbol{u}-\boldsymbol{u}_{h}\right\|_{1, \Omega}+\left\|\lambda-\theta_{h}\right\|_{-\frac{1}{2}, \Gamma_{C}} \leq C h^{\frac{1+\eta}{2}}\|\boldsymbol{u}\|_{\frac{3}{2}+\eta, \Omega},
$$

and that the proofs of Lemmas 46 remain the same when dropping the assumption (18).

Remark 4. Using the same techniques as in 21, it becomes possible to obtain the same bounds as in Theorem 1 and Corollary 1 for the error with a weighted $L^{2}$-norm on the multipliers: $\left\|\boldsymbol{u}-\boldsymbol{u}_{h}\right\|_{1, \Omega}+h_{C}^{\frac{1}{2}}\left\|\lambda-\lambda_{h}\right\|_{0, \Gamma_{C}}$.

\section{Numerical experiments}

This section is concerned with the numerical implementation of the finite element method (9) and its comparison with the standard approach (13). We suppose that the contacting bodies are homogeneous isotropic so that Hooke's law (2) becomes

$$
\boldsymbol{\sigma}(\boldsymbol{v})=\frac{E \nu}{(1-2 \nu)(1+\nu)} \operatorname{tr}(\varepsilon(\boldsymbol{v})) \boldsymbol{I}+\frac{E}{1+\nu} \varepsilon(\boldsymbol{v}),
$$

where $I$ represents the identity matrix, 'tr' is the trace operator, $E$ and $\nu$ denote Young's modulus and Poisson's ratio, respectively, with $E>0$ and $0 \leq \nu<1 / 2$. Hereafter we denote by $N_{C}$ the number of elements on the contact area $\Gamma_{C}$.

In the first test we compute the values of the standard and nonstandard multipliers $\theta_{h}$ and $\lambda_{h}$, and we discuss the convergence rate of $\left\|\lambda_{h}-\theta_{h}\right\|_{0, \Gamma_{C}}$. The second example deals with Hertzian contact where the exact multiplier $\lambda$ is known. This allows us to compare the accuracy of both discrete multipliers. A case with two contacting bodies and nonmatching meshes on the contact area is considered in the third example. We show how the sign preserving approach can be extended to this framework, at least numerically.

6.1. First example with slow variation in the contact pressure. We study a realistic physical example also considered by Hild and Nicaise (2007) (see Fig. 1). We choose the domain $\Omega=] 0,1[\times] 0,1[$, and we suppose that the body is an iron square of $1 \mathrm{~m}^{2}$ whose material characteristics are $E=2.110^{11} \mathrm{~Pa}, \nu=0.3$ and

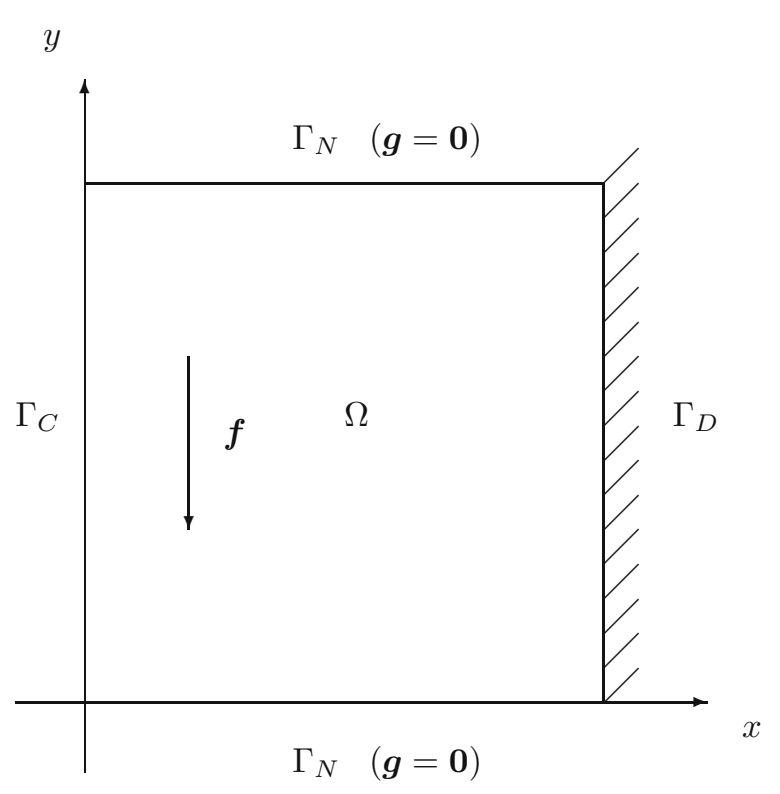

Fig. 1. Geometry of the body $\Omega$.

$\rho=7800 \mathrm{~kg} \cdot \mathrm{m}^{-3}$. The body is clamped on its right side; it is initially in contact on its left side and no forces are applied on the upper and lower boundary parts of $\Omega$. Moreover, the body is acted on by its own weight only (with $g=9.81 \mathrm{~m} \cdot \mathrm{s}^{-2}$ ). We consider quasi-uniform un-

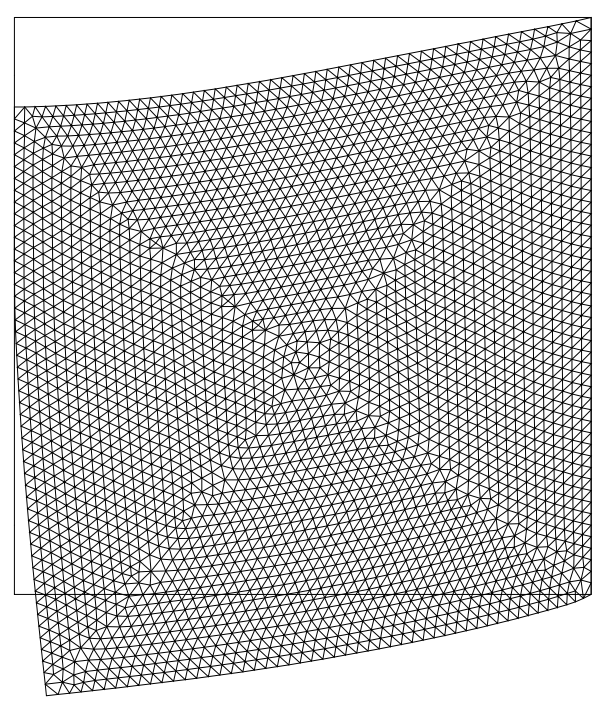

Fig. 2. Initial and deformed configuration with $N_{C}=50$ (deformation is amplified by a factor $2 \cdot 10^{5}$ ). 
structured meshes. A first configuration with 51 nodes on the contact area is depicted in Fig. 2. We see that $\Gamma_{C}$ is divided into two parts: an upper part where the body remains in contact with the axis $x=0$, and the lower part of $\Gamma_{C}$ where it separates from this axis.

The nodes on $\Gamma_{C}$ are numbered from 1 (up) to 51 (bottom), and $u_{h n}=0$ at nodes 1 to 16 whereas $u_{h n}<0$ at the other nodes. The corresponding standard (resp. nonstandard) multipliers $\theta_{h}$ (resp. $\lambda_{h}$ ) are reported in Table 1 . As expected, we observe that $\theta_{h}$ is sometimes negative and that it shows some artificial (from a mechanical point of view) oscillations on the separation part (nodes 16 to 51). These oscillations weaken when moving away from the transition point (node 16).

Table 1. Nonstandard and standard multipliers $\lambda_{h}$ and $\theta_{h}$.

\begin{tabular}{|c|c|c|c|}
\hline Node & $\lambda_{h}$ & & $\theta_{h}$ \\
\hline 1 & $1.20010 \mathrm{E}+05$ & & $1.26478 \mathrm{E}+05$ \\
\hline 2 & $1.09245 \mathrm{E}+05$ & & $1.07075 \mathrm{E}+05$ \\
\hline 3 & $1.00097 \mathrm{E}+05$ & & $1.00693 \mathrm{E}+05$ \\
\hline 4 & $9.10860 \mathrm{E}+04$ & & $9.07382 \mathrm{E}+04$ \\
\hline 5 & $8.29212 \mathrm{E}+04$ & & $8.28702 \mathrm{E}+04$ \\
\hline 6 & $7.53913 \mathrm{E}+04$ & & $7.53082 \mathrm{E}+04$ \\
\hline 7 & $6.83133 \mathrm{E}+04$ & & $6.82448 \mathrm{E}+04$ \\
\hline 8 & $6.16557 \mathrm{E}+04$ & & $6.15925 \mathrm{E}+04$ \\
\hline 9 & $5.53683 \mathrm{E}+04$ & & $5.53196 \mathrm{E}+04$ \\
\hline 10 & $4.93761 \mathrm{E}+04$ & & $4.93389 \mathrm{E}+04$ \\
\hline 11 & $4.36050 \mathrm{E}+04$ & & $4.35813 \mathrm{E}+04$ \\
\hline 12 & $3.79678 \mathrm{E}+04$ & & $3.79658 \mathrm{E}+04$ \\
\hline 13 & $3.23468 \mathrm{E}+04$ & & $3.23622 \mathrm{E}+04$ \\
\hline 14 & $2.65571 \mathrm{E}+04$ & & $2.66665 \mathrm{E}+04$ \\
\hline 15 & $2.02133 \mathrm{E}+04$ & & $2.03146 \mathrm{E}+04$ \\
\hline 16 & $1.16927 \mathrm{E}+04$ & & $1.33550 \mathrm{E}+04$ \\
\hline 17 & 0 & - & $3.57846 \mathrm{E}+03$ \\
\hline 18 & 0 & & $9.58847 \mathrm{E}+02$ \\
\hline 19 & 0 & - & $2.56922 \mathrm{E}+02$ \\
\hline 20 & 0 & & $6.88421 \mathrm{E}+01$ \\
\hline 21 & 0 & - & $1.84462 \mathrm{E}+01$ \\
\hline 22 & 0 & & $4.94264 \mathrm{E}+00$ \\
\hline 23 & 0 & - & $1.32438 \mathrm{E}+00$ \\
\hline 24 & 0 & & $3.54866 \mathrm{E}-01$ \\
\hline 25 & 0 & - & $9.50859 \mathrm{E}-02$ \\
\hline 26 & 0 & & $2.54782 \mathrm{E}-02$ \\
\hline 27 & $\overline{0}$ & - & $6.82686 \mathrm{E}-03$ \\
\hline 28 & 0 & & $1.82925 \mathrm{E}-03$ \\
\hline 29 & 0 & - & $4.90147 \mathrm{E}-04$ \\
\hline 30 & 0 & & $1.31334 \mathrm{E}-04$ \\
\hline 31 & 0 & - & $3.51910 \mathrm{E}-05$ \\
\hline 32 & 0 & & $9.42939 \mathrm{E}-06$ \\
\hline 33 & 0 & - & $2.52660 \mathrm{E}-06$ \\
\hline 34 & 0 & & $6.77000 \mathrm{E}-07$ \\
\hline 35 & 0 & - & $1.81401 \mathrm{E}-07$ \\
\hline 36 & 0 & & $4.86064 \mathrm{E}-08$ \\
\hline 37 & 0 & - & $1.30240 \mathrm{E}-08$ \\
\hline 38 & 0 & & $3.48978 \mathrm{E}-09$ \\
\hline 39 & 0 & - & $9.35084 \mathrm{E}-10$ \\
\hline 40 & 0 & & $2.50555 \mathrm{E}-10$ \\
\hline 41 & 0 & - & $6.71360 \mathrm{E}-11$ \\
\hline 42 & 0 & & $1.79890 \mathrm{E}-11$ \\
\hline 43 & 0 & - & $4.82015 \mathrm{E}-12$ \\
\hline 44 & 0 & & $1.29155 \mathrm{E}-12$ \\
\hline 45 & 0 & - & $3.46071 \mathrm{E}-13$ \\
\hline 46 & $\overline{0}$ & & $9.27297 \mathrm{E}-14$ \\
\hline 47 & $\overline{0}$ & - & $2.48474 \mathrm{E}-14$ \\
\hline 48 & 0 & & $6.66014 \mathrm{E}-15$ \\
\hline 49 & 0 & - & $1.79311 \mathrm{E}-15$ \\
\hline 50 & 0 & & $5.12319 \mathrm{E}-16$ \\
\hline 51 & 0 & - & $2.56159 \mathrm{E}-16$ \\
\hline
\end{tabular}

We then compute the convergence rate of $\| \lambda_{h}-$ $\theta_{h} \|_{0, \Gamma_{C}}$ in order to illustrate Lemma 6. The results are reported in Table 2, where this expression is computed from $N_{C}=1$ to $N_{C}=128$. The average convergence rate (between $N_{C}=8$ and $N_{C}=128$ ) is 1.25 , and a limit rate near 1.24 is observed. In this example, we avoid computing the convergence rates of $\left\|\lambda-\lambda_{h}\right\|_{0, \Gamma_{C}}$ and $\left\|\lambda-\theta_{h}\right\|_{0, \Gamma_{C}}$ since the problem (7) does not admit an explicit solution $(\lambda, \boldsymbol{u})$ in this case and the choice of a reference multiplier would require to choose one of the methods (9) or (13). This study will be performed in the next example, where the exact expression of the multiplier $\lambda$ is known. Of course, such a phenomenon does not occur for the reference displacement since they coincide for both finite element methods (9) and (13) according to Proposition 2. Thus we compute a reference displacement denoted by $\boldsymbol{u}_{\text {ref }}$ corresponding to a mesh which is as fine as possible. The most refined mesh corresponds to 129 nodes on the contact area and it furnishes the reference solution $\boldsymbol{u}_{\text {ref }}$ which is the chosen approximation for $\boldsymbol{u}$.

Table 2. Multipliers difference and error on the displacements.

\begin{tabular}{|l||l|l|}
\hline & $\left\|\lambda_{h}-\theta_{h}\right\|_{0, \Gamma_{C}}$ & $(\mathrm{a}(e, e))^{1 / 2}$ \\
\hline \hline$N_{C}=1$ & 17063 & 0.12778 \\
\hline$N_{C}=2$ & 17299 & $9.72400 \quad 10^{-2}$ \\
\hline$N_{C}=4$ & 13355 & $7.01423 \quad 10^{-2}$ \\
\hline$N_{C}=8$ & 6181.4 & $4.40570 \quad 10^{-2}$ \\
\hline$N_{C}=16$ & 2789 & $2.54805 \quad 10^{-2}$ \\
\hline$N_{C}=32$ & 1121 & $1.40710 \quad 10^{-2}$ \\
\hline$N_{C}=64$ & 453.27 & - \\
\hline$N_{C}=128$ & 191.29 & - \\
\hline Limit rate & 1.24 & 0.86 \\
\hline
\end{tabular}

We set $\boldsymbol{e}=\boldsymbol{u}_{\mathrm{ref}}-\boldsymbol{u}_{h}$. Since the limit convergence rate of $(a(\boldsymbol{e}, \boldsymbol{e}))^{1 / 2}$ (which is a norm equivalent to $\left.\left\|\boldsymbol{u}_{\mathrm{ref}}-\boldsymbol{u}_{h}\right\|_{1, \Omega}\right)$ is near 0.86 , one could merely believe that the convergence rate of $\left\|\lambda_{h}-\theta_{h}\right\|_{0, \Gamma_{C}}$ would be around 0.36 . In fact, the computed rate (of 1.25) is much higher, a phenomenon that we cannot explain.

From this example we conclude, as expected, that the multiplier $\lambda_{h}$ is more relevant from a mechanical point of view than $\theta_{h}$.

6.2. Example of Hertzian contact. The next example is concerned with the Hertzian contact problem of an elastic ball with an infinite half plane. The material characteristics of the ball of radius $r=1 \mathrm{~mm}$ are chosen as in the work of Hüeber and Wohlmuth (2005a): $\nu=0.3$, $E=7000 \mathrm{MPa}$, and a force of $(0,-f)$ with $f=100 \mathrm{~N}$ is applied at the top of the ball. Since the analytical expression of the contact pressure is

$$
\lambda(x)=\frac{2 f}{\pi b^{2}} \sqrt{b^{2}-x^{2}}, \quad-b \leq x \leq b,
$$




$$
b=2 \sqrt{\frac{f r\left(1-\nu^{2}\right)}{E \pi}},
$$

we have at our disposal a useful analytical solution for a comparison of $\lambda_{h}$ and $\theta_{h}$. Here $b \approx 0.1286 \mathrm{~mm}$ and $\lambda(x) \approx 494.8 \sqrt{1-(x / b)^{2}} \mathrm{~N} / \mathrm{mm},-b \leq x \leq b$. In our computations we choose quasi-uniform unstructured meshes (we do not symmetrize the problem and the mesh is not symmetric). The results are reported in Tables 3 and 4 .

Table 3. Errors on the multipliers.

\begin{tabular}{|c||c|c|}
\hline Nodes on $\partial \Omega$ & $\left\|\lambda-\lambda_{h}\right\|_{0, \Gamma_{C}}$ & $\left\|\lambda-\theta_{h}\right\|_{0, \Gamma_{C}}$ \\
\hline \hline 24 & 47.080 & 100.23 \\
\hline 48 & 54.651 & 80.604 \\
\hline 96 & 23.704 & 30.822 \\
\hline 192 & 8.9620 & 15.223 \\
\hline 384 & 1.8805 & 9.8732 \\
\hline 768 & 1.2057 & 4.4893 \\
\hline Average rate & 1.057 & 0.896 \\
\hline
\end{tabular}

Table 4. Comparison of the multipliers.

\begin{tabular}{|c||l|l|}
\hline $\begin{array}{c}\text { Nodes on } \\
\partial \Omega\end{array}$ & $\begin{array}{l}\max _{\Gamma_{C}} \lambda_{h} ; \\
\min _{\Gamma_{C}} \lambda_{h}\end{array}$ & $\begin{array}{l}\max _{\Gamma_{C}} \theta_{h} ; \\
\min _{\Gamma_{C}} \theta_{h}\end{array}$ \\
\hline \hline 24 & $381.97 ; 0$ & $663.98 ;-178.76$ \\
\hline 48 & $511.47 ; 0$ & $769.27 ;-3.0305$ \\
\hline 96 & $503.82 ; 0$ & $535.93 ;-19.902$ \\
\hline 192 & $498.20 ; 0$ & $501.49 ;-40.091$ \\
\hline 384 & $496.93 ; 0$ & $498.27 ;-59.165$ \\
\hline 768 & $496.43 ; 0$ & $496.77 ;-45.345$ \\
\hline
\end{tabular}

We first observe that the convergence rates of $\| \lambda-$ $\lambda_{h} \|_{0, \Gamma_{C}}$ and $\left\|\lambda-\theta_{h}\right\|_{0, \Gamma_{C}}$ are not constant when $h$ decreases: the average rates are 1.057 and 0.896 , respectively, so that the terms $\left\|\lambda-\lambda_{h}\right\|_{0, \Gamma_{C}}$ remain smaller than $\left\|\lambda-\theta_{h}\right\|_{0, \Gamma_{C}}$ as $h$ vanishes. From the expression (22), we see that $\left(\max _{\Gamma_{C}} \lambda, \min _{\Gamma_{C}} \lambda\right)$ is approximately $(494.8,0)$. The first argument is reached by the two approaches, but the value 0 is not obtained in a satisfactory way by $\theta_{h}$.

From this example we conclude that the sign preserving approach involving the nonnegative multiplier $\lambda_{h}$ is more accurate than the standard method.

6.3. Example with two contacting bodies and nonmatching meshes. As the third example we choose a problem of two contacting bodies $\Omega^{1}$ and $\Omega^{2}$ with nonmatching meshes on the common contact zone $\Gamma_{C}=$ $\overline{\Omega^{1}} \cap \overline{\Omega^{2}}$. The dimensions of $\Omega^{1}$ and $\Omega^{2}$ are $1 \mathrm{~mm} \times$ $0.05 \mathrm{~mm}$. Poisson's ratio $\nu=0.2$ for both solids, Young's modulus $E_{1}=13000 \mathrm{MPa}$ for the upper body, and $E_{2}=30000 \mathrm{MPa}$ for the lower body are assumed. There are two applied boundary loads on $\Omega^{1}$, of a value of $100 \mathrm{~N} / \mathrm{mm}$ (see Fig. 3): $\boldsymbol{g}_{1}$ (on the upper half of the left side) and $\boldsymbol{g}_{2}$ (on the right half of the upper side). Symmetry conditions are applied on the lower and right parts of the structure. The mesh of $\Omega^{1}$ (resp. $\Omega^{2}$ ) divides $\Gamma_{C}$ into 119 (resp. 120) identical segments.

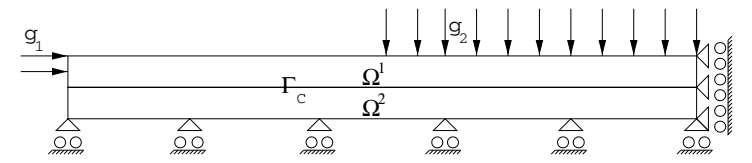

Fig. 3. Setting of the problem.

In order to handle nonmatching meshes, we consider a global contact condition of a mortar type. For error estimates dealing with mortar methods for contact problems, we refer the reader to, e.g., the works of Ben Belgacem et al. (1999), Coorevits et al. (2002), Hild (2000), Hüeber and Wohlmuth (2005a; 2005b), and Wohlmuth and Krause (2003). Such a contact condition furnishes a multiplier denoted $\theta_{h}^{1}$ which does not satisfy the nonnegativity condition. Our aim is to extend, at least numerically, the range of applicability of the sign preserving method to a configuration with nonmatching meshes.

We denote by $\boldsymbol{V}_{h}^{1}$ and $\boldsymbol{V}_{h}^{2}$ the finite element spaces associated with $\Omega^{1}$ and $\Omega^{2}$, and by $M_{h}^{1+}$ the positive polar cone of $W_{h}^{1+}$ (see the definition in (14)). Note that the set $W_{h}^{1+}$ involves functions defined on $\Gamma_{C}$ which are continuous, nonnegative and piecewise of degree one on the mesh of $\Omega^{1}$. Of course, one could also choose a symmetrical definition (e.g., $M_{h}^{2+}$ ) using the mesh of $\Omega^{2}$. The standard approach is to find $\boldsymbol{u}_{h}=\left(\boldsymbol{u}_{h}^{1}, \boldsymbol{u}_{h}^{2}\right) \in \boldsymbol{V}_{h}^{1} \times \boldsymbol{V}_{h}^{2}$ and $\theta_{h}^{1} \in M_{h}^{1+}$ satisfying (see Coorevits et al., 2002; Hüeber and Wohlmuth, 2005a)

$$
\left\{\begin{array}{c}
a\left(\boldsymbol{u}_{h}, \boldsymbol{v}_{h}\right)+\int_{\Gamma_{C}} \theta_{h}^{1}\left(v_{h n}^{1}+v_{h n}^{2}\right) \mathrm{d} \Gamma=L\left(\boldsymbol{v}_{h}\right), \\
\forall \boldsymbol{v}_{h} \in \boldsymbol{V}_{h}^{1} \times \boldsymbol{V}_{h}^{2}, \\
\int_{\Gamma_{C}}\left(\mu_{h}^{1}-\theta_{h}^{1}\right)\left(u_{h n}^{1}+u_{h n}^{2}\right) \mathrm{d} \Gamma \leq 0, \forall \mu_{h}^{1} \in M_{h}^{1+},
\end{array}\right.
$$

where $a(\cdot, \cdot)$ and $L(\cdot)$ denote respectively the bilinear and linear forms involving both bodies $\Omega^{1}$ and $\Omega^{2}$. The sign preserving approach is to find $\boldsymbol{u}_{h}=\left(\boldsymbol{u}_{h}^{1}, \boldsymbol{u}_{h}^{2}\right) \in \boldsymbol{V}_{h}^{1} \times \boldsymbol{V}_{h}^{2}$ and $\lambda_{h}^{1} \in W_{h}^{1+}$ satisfying

$$
\left\{\begin{array}{c}
a\left(\boldsymbol{u}_{h}, \boldsymbol{v}_{h}\right)+\int_{\Gamma_{C}} I_{h}^{1}\left(\lambda_{h}^{1}\left(v_{h n}^{1}+p_{h}^{1}\left(v_{h n}^{2}\right)\right)\right) \mathrm{d} \Gamma=L\left(\boldsymbol{v}_{h}\right), \\
\forall \boldsymbol{v}_{h} \in \boldsymbol{V}_{h}^{1} \times \boldsymbol{V}_{h}^{2}, \\
\int_{\Gamma_{C}} I_{h}^{1}\left(\left(\mu_{h}^{1}-\lambda_{h}^{1}\right)\left(u_{h n}^{1}+p_{h}^{1}\left(u_{h n}^{2}\right)\right)\right) \mathrm{d} \Gamma \leq 0, \\
\forall \mu_{h}^{1} \in W_{h}^{1+},
\end{array}\right.
$$

where $I_{h}^{1}$ denotes the Lagrange interpolation operator of degree 1 at the nodes of $\Omega^{1}$ on $\Gamma_{C}$, and $p_{h}^{1}$ stands for the $L^{2}\left(\Gamma_{C}\right)$-projection operator onto $W_{h}^{1}$ (see $\underline{20}$ ).

As expected, the deformed configuration shows a separation area on the left part of $\Gamma_{C}$ and a contact area on the right part of $\Gamma_{C}$ (see Fig. 4). The multiplier $\theta_{h}^{1}$, representing the contact pressure is depicted in Fig. 5. As 
already noticed, the multiplier is not always nonnegative and it shows some artificial oscillations near the transition point from contact to separation. Besides, the multiplier $\lambda_{h}^{1}$ is represented in Fig. 6 and we observe that it is more relevant from a mechanical point of view. We observe that the multiplier value is close to 100 on the contact zone which corresponds to the value of $\boldsymbol{g}_{2}$. Finally, the difference $\theta_{h}^{1}-\lambda_{h}^{1}$ is depicted in Fig. 7 and we see that $\theta_{h}^{1}$ and $\lambda_{h}^{1}$ differ in a significant way near the transition point. Again, we conclude that the new approach involving $\lambda_{h}^{1}$ seems to be more accurate than the standard one when handling nonmatching meshes.

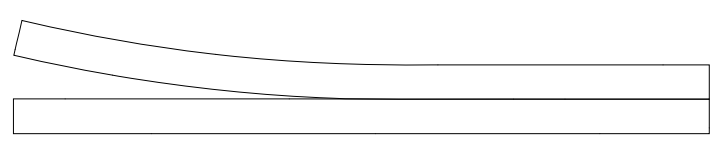

Fig. 4. Deformed configuration.

\section{Conclusion}

In this work we consider a mixed finite element method which furnishes primal and dual variables with a good sign in opposition to the already known mixed methods for contact problems (in particular, the classical approach). The study of the method uses an averaging positivity preserving operator, which is analyzed and discussed. The convergence analysis in this paper leads to the same error estimates as the standard approach. The numerical experiments obtained with the new method seem to be more relevant and efficient in comparison with the standard method. Finally, the friction (see, e.g., Hild, 2002) or the crack problems (see, e.g., Belhachmi et al., 2005; Khludnev and Sokolowski, 2004) are some possible applications of the method.

\section{References}

Adams, R. (1975). Sobolev Spaces, Academic Press, New York, NY/London.

Belhachmi, Z., Sac-Epée, J.-M. and Sokolowski, J. (2005). Mixed finite element methods for smooth domain formulation of crack problems, SIAM Journal on Numerical Analysis 43(3): 1295-1320.

Ben Belgacem, F. and Brenner, S. (2001). Some nonstandard finite element estimates with applications to 3D Poisson and Signorini problems, Electronic Transactions on Numerical Analysis 12: 134-148.

Ben Belgacem, F., Hild, P. and Laborde, P. (1999). Extension of the mortar finite element method to a variational inequality modeling unilateral contact, Mathematical Models and Methods in the Applied Sciences 9(2): 287-303.

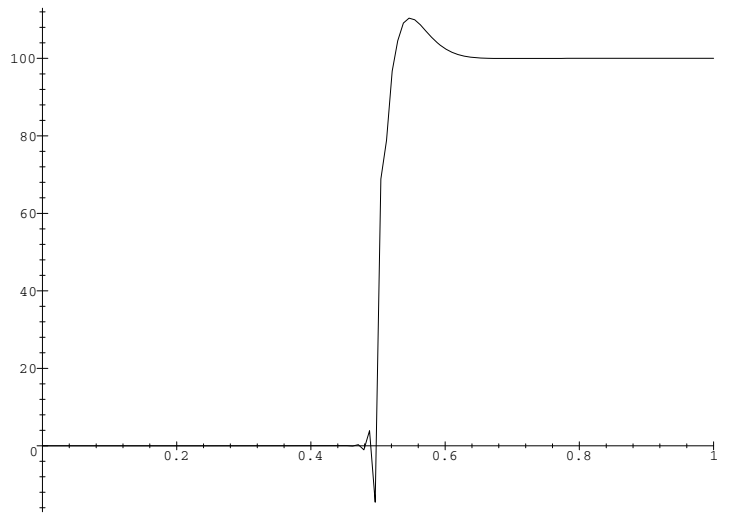

Fig. 5. Multiplier $\theta_{h}^{1}$.

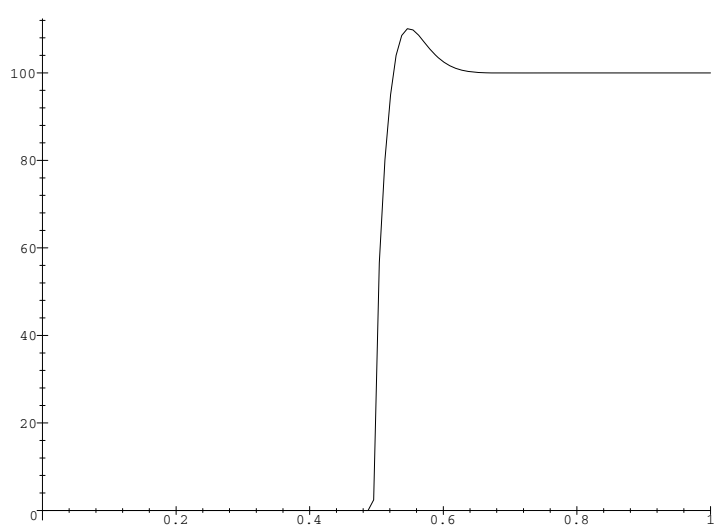

Fig. 6. Multiplier $\lambda_{h}^{1}$.

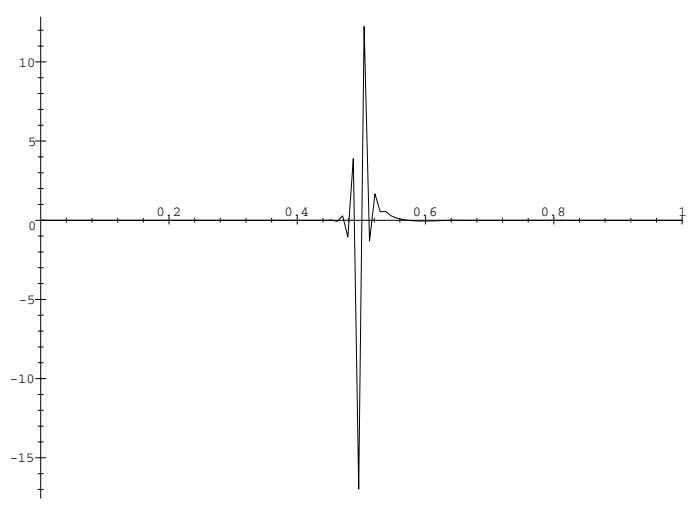

Fig. 7. Difference between the multipliers: $\theta_{h}^{1}-\lambda_{h}^{1}$. 
Ben Belgacem, F. and Renard, Y. (2003). Hybrid finite element methods for the Signorini problem, Mathematics of Computation 72(243): 1117-1145.

Bernardi, C. and Girault, V. (1998). A local regularisation operator for triangular and quadrilateral finite elements, SIAM Journal on Numerical Analysis 35(5): 1893-1916.

Brenner, S. and Scott, L. (2002). The Mathematical Theory of Finite Element Methods, Springer-Verlag, New York, NY.

Chen, Z. and Nochetto, R. (2000). Residual type a posteriori error estimates for elliptic obstacle problems, Numerische Mathematik 84(4): 527-548.

Ciarlet, P. (1991). The finite element method for elliptic problems, in P.G. Ciarlet and J.-L. Lions (Eds.), Handbook of Numerical Analysis, Vol. II, Part 1, North Holland, Amsterdam, pp. 17-352.

Clément, P. (1975). Approximation by finite element functions using local regularization, RAIRO Modélisation Mathématique et Analyse Numérique 2(R-2): 77-84.

Coorevits, P., Hild, P., Lhalouani, K. and Sassi, T. (2002). Mixed finite element methods for unilateral problems: Convergence analysis and numerical studies, Mathematics of Computation 71(237): 1-25.

Duvaut, G. and Lions, J.-L. (1972). Les inéquations en mécanique et en physique Dunod, Paris.

Eck, C., Jarušek, J. and Krbec, M. (2005). Unilateral Contact Problems. Variational Methods and Existence Theorems, CRC Press, Boca Raton, FL.

Fichera, G. (1964). Elastic problems with unilateral constraints, the problem of ambiguous boundary conditions, Memorie della Accademia Nazionale dei Lincei 8(7): 91-140, (in Italian).

Fichera, G. (1974). Existence theorems in linear and semilinear elasticity, Zeitschrift für Angewandte Mathematik und Mechanik 54(12): 24-36.

Grisvard, P. (1985). Elliptic Problems in Nonsmooth Domains, Pitman, Boston, MA.

Han, W. and Sofonea, M. (2002). Quasistatic Contact Problems in Viscoelasticity and Viscoplasticity, American Mathematical Society, Providence, RI.

Haslinger, J., Hlaváček, I. and Nečas, J. (1996). Numerical methods for unilateral problems in solid mechanics, in P. Ciarlet and J.-L. Lions (Eds.), Handbook of Numerical Analysis, Vol. IV, Part 2, North Holland, Amsterdam, pp. 313-485.

Hilbert, S. (1973). A mollifier useful for approximations in Sobolev spaces and some applications to approximating solutions of differential equations, Mathematics of Computation 27: 81-89.

Hild, P. (2000). Numerical implementation of two nonconforming finite element methods for unilateral contact, Computer Methods in Applied Mechanics and Engineering 184(1): 99-123.
Hild, P. (2002). On finite element uniqueness studies for Coulomb's frictional contact model, International Journal of Applied Mathematics and Computer Science 12(1): 4150 .

Hild, P. and Nicaise, S. (2007). Residual a posteriori error estimators for contact problems in elasticity, Mathematical Modelling and Numerical Analysis 41(5): 897-923.

Hiriart-Urruty, J.-B. and Lemaréchal, C. (1993). Convex Analysis and Minimization Algorithms I, Springer, Berlin.

Hüeber, S. and Wohlmuth, B. (2005a). An optimal error estimate for nonlinear contact problems, SIAM Journal on Numerical Analysis 43(1): 156-173.

Hüeber, S. and Wohlmuth, B. (2005b). A primal-dual active set strategy for non-linear multibody contact problems, Computer Methods in Applied Mechanics and Engineering 194(27-29): 3147-3166.

Khludnev, A. and Sokolowski, J. (2004). Smooth domain method for crack problems, Quarterly of Applied Mathematics 62(3): 401-422.

Kikuchi, N. and Oden, J. (1988). Contact Problems in Elasticity, SIAM, Philadelphia, PA.

Laursen, T. (2002). Computational Contact and Impact Mechanics, Springer, Berlin.

Nochetto, R. and Wahlbin, L. (2002). Positivity preserving finite element approximation, Mathematics of Computation 71(240): 1405-1419.

Scott, L. and Zhang, S. (1990). Finite element interpolation of nonsmooth functions satisfying boundary conditions, Mathematics of Computation 54(190): 483-493.

Strang, G. (1972). Approximation in the finite element method, Numerische Mathematik 19: 81-98.

Wohlmuth, B. and Krause, R. (2003). Monotone multigrid methods on nonmatching grids for nonlinear multibody contact problems, SIAM Journal on Scientific Computation 25(1): 324-347.

Wriggers, P. (2002). Computational Contact Mechanics, Wiley, Chichester.

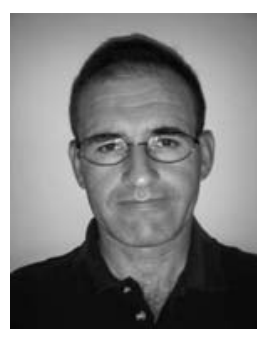

Patrick Hild received the Ph.D. degree in applied mathematics from the University of Toulouse in 1998. He worked as an assistant professor at the Savoy University in Chambéry, France, in the years 1998-2002. In 2002, he joined Franche-Comté University in Besançon, France, as a full professor. His research areas cover various topics in the modeling and numerical simulation of solid mechanics, numerical analysis, contact and friction problems.

Received: 8 November 2010

Revised: 30 January 2011

Re-revised: 21 March 2011 